Portland State University

PDXScholar

Fall 2020

\title{
The Utility of Multiplex Closeness Centrality for Predicting Item Difficulty Parameters in Anomia Tests
}

Khanh L. Nguyen

Portland State University

Follow this and additional works at: https://pdxscholar.library.pdx.edu/honorstheses

Part of the Speech and Hearing Science Commons

Let us know how access to this document benefits you.

\section{Recommended Citation}

Nguyen, Khanh L., "The Utility of Multiplex Closeness Centrality for Predicting Item Difficulty Parameters in Anomia Tests" (2020). University Honors Theses. Paper 939.

https://doi.org/10.15760/honors.962

This Thesis is brought to you for free and open access. It has been accepted for inclusion in University Honors Theses by an authorized administrator of PDXScholar. Please contact us if we can make this document more accessible: pdxscholar@pdx.edu. 


\title{
The Utility of Multiplex Closeness Centrality for Predicting Item Difficulty Parameters in
}

\author{
Anomia Tests \\ Khanh L. Nguyen \\ Department of Speech and Hearing Sciences, Portland State University \\ Dr. Gerasimos Fergadiotis
}

November 20, 2020 


\begin{abstract}
Background: Confrontation naming tests for the assessment of aphasia are perhaps the most commonly used tests in aphasiology. Recently, such tests have been modeled using item response theory approaches. Despite their advantages, item response theory models require large sample sizes for parameter estimation that are often unrealistic when working with clinical populations. As an alternative approach, Fergadiotis, Kellough \& Hula (2015) explored automatic item calibration by regressing item difficulty parameters on word length, age of acquisition (AOA; Kuperman, Stadthagen-Gonzalez, \& Brysbaert, 2012), and lexical frequency as quantified by the Log10CD index (Brysbaert \& New, 2009). Despite the high predictive utility that they achieved, the model's performance was far from perfect $\left(R^{2}=.63\right)$ which may carry implications for the accuracy of any difficulty parameters derived by the model.

Purpose: This study aims to examine the addition of a fourth psycholinguistic variable to the regression model, multiplex closeness centrality (MCC) (Castro \& Stella, 2019). It is hypothesized that the ability to capture how well-connected words are in the human lexicon would make MCC a potential indicator of semantic processing which would contribute to the predictive utility of the model.
\end{abstract}

Method: A multiple regression analysis was carried out with the Philadelphia Naming Test item difficulty parameters as the dependent variable, and lexical frequency, AOA, word length, and MCC as the predictors. Item difficulty parameters were estimated based on a traditional calibration approach (Fergadiotis, et al., 2015).

Results \& Conclusions: Our analysis showed a high correlation between MCC and item difficulty and suggested that the addition of MCC has allowed the model to account for more variance. However, the change between the model with three variables and the one with four 
variables, including MCC, was not statistically significant. In other words, MCC did not add unique information to the regression model despite the high correlation with item difficulty due to the overlapping variance of MCC with other predictors. However, the findings should be interpreted cautiously because of a large number of missing values in MCC. Post hoc analyses indicated that data were missing not at random which might have contributed to the lack of significant findings. Thus, we suggest that future research investigate this type of study using a complete dataset and appropriately apply the missing data theory to their analysis. 
Aphasia is an acquired neurogenic language disorder, often the result of a stroke, that affects more than 2.4 million people in the US (Simmons-Mackie, 2018). Typically, people with aphasia (PWA) present with anomia, which refers to the inability to access and retrieve words during language production (Goodglass \& Wingfield, 1997; Raymer \& Rothi, 2012). Further, PWA exhibit symptoms of anomia even when other symptoms of aphasia resolve during the evolution of a stroke (e.g., morphosyntactical deficits). Since anomia is the cardinal deficit in aphasia, anomia treatment has received considerable attention in aphasiology. Further, given that anomia is a primary diagnostic feature of aphasia, clinicians typically include evaluations of word access and retrieval in the batteries of tests administered to stroke patients.

Perhaps the most commonly used tool to assess anomia both in research and clinical contexts are confrontation naming tests (CNTs) (Brady et al., 2016; Kiran et al., 2018). These tests consist of illustrations of common objects. Typically, an item is administered and PWA are evaluated as they name the items. Beside their clinical usage, the CNTs have served as a fundamental tool in many aphasiology-related research studies. Particularly, Dell and his colleagues have developed a model of the cognitive machinery underlying word production based on people's performances on CNTs (G. Dell et al., 1997; Schwartz et al., 2006). Error types retrieved from CNTs have also contributed to lesion analyses, which are used to study the neural correlates of language deficits (Schwartz et al., 2009). Moreover, researchers have used these CNTs to verify the efficacy of different treatment approaches (Kendall et al., 2015; Quique et al., 2019) and the cortical reorganization after anomia treatment (Fridriksson et al., 2006).

There is a long history of developing such tests, and different CNTs have been created throughout the past few decades by different test developers and speech language pathologists. Some of the most frequently used ones are the Philadelphia Naming Test (PNT) (Roach et al., 
1996), the Boston Naming Test (Kaplan et al., 1983), and the Snodgrass and Vanderwart stimuli (Snodgrass \& Vanderwart, 1980). Moreover, all major aphasia batteries have a naming subtest, as seen in the Western Aphasia Battery - R (Kertesz, 2007), the Comprehensive Aphasia Test (Swinburn et al., 2004), the Boston Diagnostic Aphasia Examination (Goodglass \& Kaplan, 1972), the Preliminary Neuropsychological Battery (Cossa et al., 1999), and the Object and Action Naming Battery (Druks \& Masterson, 2000).

Despite the popularity of confrontation picture naming tests, their utility for quantifying anomia is limited by at least four issues. First, patients' ability estimates collected from different tests are placed on different metrics and cannot be directly compared (Fergadiotis, Swiderski, et al., 2019). The difficulty stems in part from the varied difficulty of the items being used. For instance, a $20 \%$ accuracy on a test with difficult words (e.g., stethoscope) may not necessarily indicate worse naming ability than the $30 \%$ accuracy on a test with easier words (e.g., cat). This prevents the direct comparison of estimates across CNTs which: (i) disrupts the flow of clinical information across healthcare settings that use different CNTs; (ii) may lead to unnecessary testing of patients depending on the availability of CNTs at each setting; and (iii) restricts our ability to conduct meta-analytic studies.

Further, currently available tests invalidly assume constant measurement error based on which $95 \%$ confidence intervals are estimated around ability scores. With the exception of recent work (e.g., Fergadiotis, Hula, et al., 2019; Hula et al., 2020) on the PNT, naming tests assume equal measurement error regardless of ability level. This ignores that measurement error varies as a function of the degree to which the difficulty of the test targets the ability level of the person being tested (Embretson \& Reise, 2000). Assuming an average constant measurement error leads to invalidly narrow confidence intervals for PWA in the extremes, and overly wide confidence 
intervals for PWA in the middle of the ability distribution. Further, the assumption of equal measurement error has significant implications for the assessment of change. For example, the PNT short forms published by Walker and Schwartz (2012) are optimally targeted to, and thus most precise for PWA with moderate anomia. If one assumes a constant measurement error, then any confidence intervals and associated probabilities derived about the change score may be distorted. The confidence intervals around change score estimates for very mildly and severely impaired PWA may be misleadingly narrow, leading to an inflated type I error rate. On the other hand, the width of confidence intervals around change scores for moderately impaired PWA may be overestimated, leading to decreased power to detect real change and an increased type II error rate.

Another notable limitation of currently available tests is that they are inefficient. Particularly, most tests must be administered in their entirety, leading to long administration times and increased testing burden for clinicians and patients. In addition, the items are a priori selected and as a result, they could either be too challenging or too easy for certain test takers. Therefore, a patient may experience frustration or boredom the longer the test is carried out, which may affect their performance and contaminate the test scores.

Finally, with limited exceptions (Hula et al., 2020), there is a lack of tools that can generate multiple equivalent test forms with non-overlapping item content. As a result, oftentimes in practice, the same set of testing items are used throughout the course of a treatment, creating a possibility for test practice effects to influence the patients' performance leading to invalid conclusions. For example, a patient's score on the naming task may appear as improved, or may even reach the benchmark of the treatment plan, when in fact, results are due to familiarization with the testing items as opposed to effective treatment. Potentially, the 
clinician can move on to working on other conditions, leaving the naming deficit unaddressed.

Or, a patient may be told that they have improved based on their test scores, while they may not experience any actual gains to words beyond the ones included in the test.

\section{Computerized Adaptive Version of the Philadelphia Naming Test}

To address these limitations, recent studies have looked into possible improvements to CNTs. Specifically, psychometric research has focused on the Philadelphia Naming Test which is among the most commonly used CNTs in research applications (G. S. Dell, 1986). This test contains 175 items, depicted by black-and-white drawings of simple objects. All targets are nouns that range from 1-4 syllables in length. The items include high-, medium-, or lowfrequency targets as determined by Francis and Kučera (1982). Further, the items were selected from a set of 277 items that were correctly identified by $85 \%$ of a control group (Roach et al., 1996). Patients are asked to name the item that appears on the screen and their first complete response to that item, according to the PNT guidelines, is recorded. Following that, productions can go through a two-level coding system, in which their semantic relationship and phonological similarity to the targets are examined. Subsequently, erroneous productions can be assigned a code accordingly (e.g., semantic, formal, mixed, unrelated, neologism and other).

The PNT is a prominent test choice in measuring lexical retrieval ability thanks to its robust psychometric properties (Fergadiotis \& Wright, 2016; Walker \& Schwartz, 2012). The PNT's high test-retest reliability and moderate to high intercorrelations suggest high construct validity. Secondly, because the targets are known and the test takers are instructed to produce single word responses only, the ambiguity when scoring is minimized. Further, the PNT provides very specific instructions for identifying the first complete attempt and how it should be scored allowing the administrator to consistently determine whether a successful retrieval attempt has 
been achieved, and minimize construct irrelevant variance stemming from the raters (Roach et al., 1996). Another important property of the PNT that sets it apart from many other clinical diagnostic measures is its high correlation with naming impairment severity and weak correlation with demographic variables. Moreover, the testing materials are available on the Moss Rehabilitation Research Institute website, making the test much more accessible for clinical use as well as for research purposes.

To refine the PNT, recent efforts have focused on modeling its items using modern psychometrics. Specifically, Fergadiotis, Kellough, and Hula (2015) re-analyzed the PNT using item response theory (IRT) (Lord et al., 1968), a psychometric framework commonly used in educational and psychological test and scale development. Specifically, they fit a 1-parameter logistic (1-PL) model to responses from 276 PWA on the PNT. After ensuring the model's assumptions were met (e.g., unidimensionality, conditional independence) they derived the difficulty parameters for each PNT item. In addition, to investigate the construct validity of the PNT, Fergadiotis et al. also tested whether psycholinguistic variables, that based on cognitive theory were expected to predict the difficulty of different PNT items, were indeed predictive empirically. Following that, a computer adaptive version of the PNT (PNT-CAT), employing the 1-PL IRT model was developed (Hula et al., 2015) and validated via real data simulations. Based on the results, Hula et al. demonstrated that it is possible to reduce testing burden by creating shorter test forms, while minimizing the loss of precision associated with the administration of a small number of items. The computer adaptive test engine was further validated in two follow up empirical studies. Specifically, Fergadiotis, Hula, Swiderski, Lei, and Kellough ( 2019) demonstrated that scores on the PNT -CAT from 47 PWA were in high agreement with their 
scores on the full PNT. In addition, Hula et al. (2020) developed two equivalent, dynamic, test forms with non-overlapping items for repeated administrations.

In general, an IRT model focuses on predicting the response on each item of a CNT as a function of two latent variables, the patient's ability level and the difficulty parameter of each item (Ayala, 2013). In other words, given the item difficulty parameters, the IRT-based CAT engine can convert a person's observed responses to the test's items into a standardized metric that does not depend on which specific items were administered. Thus, IRT item difficulty parameters are extremely important for the computer adaptive test engine. Hula and his colleagues were able to estimate the difficulty parameters by calibrating the PNT using archival data from more than 250 PWA from the Moss Aphasia Psycholinguistic Project Database (MAPPD) (Hula et al., 2015). However, in aphasiology, collecting data from such large samples sizes is often not feasible, making the calibration of other CNTs using the same method impractical in terms of time, cost and effort.

\section{Algorithmic Estimation of Difficulty Parameter}

To overcome this barrier, Fergadiotis, Swiderski, et al. (2019) proposed an alternative approach based on which it might be possible to automatically calibrate items (i.e., estimate their difficulty) using an algorithmic estimation that does not require large sample sizes of PWA. Specifically, Fergadiotis, Swiderski, et al. (2019) identified a set of psycholinguistic variables for this purpose, consisting of word length, age of acquisition (AOA) (Kuperman et al., 2012) and lexical frequency (Brysbaert \& New, 2009). Using this combination of predictors, they developed a regression equation that can predict item difficulty parameters with relatively high accuracy. 
However, Fergadiotis et al. argued that in terms of prominent models of word production (e.g, G. S. Dell, 1986), the regression equation might not be adequately capturing how difficult a word might be in terms of semantic processing. There is general consensus (G. S. Dell, 1986; Levelt et al., 1999) that word production is a two-stage process. The first stage, lexical-semantic processing, involves the activation of semantic features and lemma selection. Then, speech sounds, or the phonological form of the word is constructed in the second stage, called the phonological processing stage.

Regarding the aforementioned three psycholinguistic variables, word length is considered primarily indicative of the second stage of lexical retrieval. However, there are conflicting hypotheses about how the remaining predictors would map onto a two-stage model of word production (Fergadiotis, Swiderski, et al., 2019). Both AOA and lexical frequency have been argued to have strong effects on either the first layer, semantic processing, the second one, phonological processing, or both. According to Kittredge and his colleagues (2008), both levels in the network are sensitive to lexical frequency, whereas AOA would have a stronger effect on the second stage. Therefore, it is likely that any indicators of how difficult a word is in terms of semantic processing could contribute additional predictive information.

\section{Multiplex Closeness Centrality}

The study of the human lexicon is crucial in learning how one acquires and retrieves words. To construct the representation of the human lexicon and to better understand its organization, in the field of network science, researchers develop language networks using corpora and behavioral language performance. In this approach, words are represented by nodes, and the relationships between words, such as semantics, syntax, phonology, orthography, are demonstrated using edges. Early literature focused on studying single layer networks separately 
(e.g. semantic network), found that semantic and phonological networks are important factors in word retrieval of both typical speakers and PWA (Castro \& Stella, 2019). However, single layer networks cannot account for the relationships among nodes in different layers of the whole network. An alternative is global network measures, one of which is closeness centrality, a measure that stands for the average inverse distance from one node to all other nodes in the network (Newman, 2010). These global network measures are known for being able to capture the interactive nature between the semantic and phonological stages, which is critical to understanding the word retrieval process in a naming task.

In the Dell's connectionist model, nodes representing concepts, lemmas and phonemes are connected through two stages: lemma selection and phonological access, where bi-directional interactions occur between concepts and lemmas; lemmas and phonemes, respectively. On the other hand, the multiplex network (Castro \& Stella, 2019) allows for connections between lemma nodes through multiple semantic and phonological network layers, or edges, namely free associations, synonyms, taxonomic relations and phonological similarities. This network, by utilizing the closeness centrality measure mentioned above, can quantify how closely nodes that represent words in the lexicon are connected to each other across layers. Closeness centrality reflects the shortest paths that connect one node to all other nodes in the network and the higher multiplex closeness centrality a node has, the easier it is to be retrieved. In other words, when a node is centrally located in the cluster of nodes or when it has the shortest connections to other nodes in the cluster, it receives the most spreading activation, which increases its likelihood to be retrieved.

\section{Purpose of the Study}


The purpose of this paper is to investigate whether a fourth psycholinguistic variable, multiplex closeness centrality (MCC) (Castro \& Stella, 2019) could be used to increase the explanatory power of the model. Generally, MCC captures how well-connected words are in the human lexicon, making it a potential indicator of semantic processing. It is hypothesized that MCC would allow us to improve the utility of the regression equation for predicting difficulty parameters, and therefore offer an alternative to traditional calibration for expanding the CNT item bank. This in turn has the potential to transform the evaluation of experimental treatment approaches and the ability of clinicians to quantify response to intervention.

\section{Method}

\section{Predictive Modeling of Item Difficulty}

We conducted a multiple regression analysis in IBM SPSS Statistics version 26 with PNT item difficulty as the dependent variable and lexical frequency, as measured by the Log $10 \mathrm{CD}$ index (Brysbaert \& New, 2009), AOA (Kuperman et al., 2012), word length, and closeness centrality as independent variables (Castro \& Stella, 2019). The predictors were entered into the regression using the enter approach for the main analysis and the forward method for the post hoc analysis. The alpha level chosen for this study in determining significance is .05 .

Dependent Variable. The PNT item difficulty parameters are the ones estimated by Fergadiotis and colleagues (Fergadiotis et al., 2015) from the sample of 251 PWA included in MAPPD (Mirman et al., 2010).

Independent Variables (Predictors). Four explanatory variables were used to predict item difficulty parameters. First, we included lexical frequency (Log10CD) norms associated with each target identified from the Subtlex corpus (Brysbaert \& New, 2009). These norms were estimated from a corpus of 51.0 million words which were gathered from the subtitles of 8,388 
films and television episodes (Brysbaert \& New, 2009). Log10CD was chosen over word form frequency from Subtlex based on evidence that Log10CD demonstrated higher explanatory utility across different experimental paradigms of psychological phenomena (Brysbaert \& New, 2009, p. 985). Further, AOA (Kuperman et al., 2012) and the number of phonemes were used. Finally, we included the multiplex closeness centrality parameter (Castro \& Stella, 2019).

\section{Preliminary Analysis}

Before conducting the regression analysis, data were evaluated to ensure there were no gross violations of the statistical analysis' assumptions. PNT item difficulty parameters and Log10CD, AOA, and length values were retrieved from Fergadiotis et al. (2015). First, linearity and homoscedasticity were checked using scatterplots. Then, we assessed the data for univariate and multivariate outliers using z-score and Mahalanobis distance, respectively. The Kolmogorov-Smirnov test was used to check the assumption of normality by testing whether the residuals were normally distributed. In addition, variance inflation factor values were used to assess multicollinearity. Finally, we explored whether missing data were present in the dataset, and we attempted to characterize the missingness by looking at the pattern among the missing values.

\section{Results}

\section{Preliminary Analysis}

According to the scatterplots shown in Figure 1, no violation of the assumptions of homoscedasticity and linearity was noted. The variability of all four psycholinguistic variables remain constant as item difficulty increases. Moderate correlations between the dependent and independent variables were also observed. Particularly, the item difficulty parameter increases as the word is learned later in life, has more phonemes, and decreases if the word is used less 
frequently, and has a lower MCC measure. The correlation coefficients between these variables are shown in Table 1. In addition, univariate outliers were identified from the examination of both the variables' z-scores and histograms (Figure 2). Subsequently, number of phonemes variable was found to have 3 univariate outliers, followed by 2 and 1 outliers in the MCC and AOA variables, respectively. Furthermore, among the univariate outliers, item numbered 86 (i.e. helicopter) has the potential to be the multivariate outlier, as seen from the Mahalanobis's distance test. The Kolmogorov-Smirnov test was also carried out to examine the normality in the distributions of these variables. MCC, AOA, lexical frequency and item difficulty were noted to be normally distributed. The distribution of number of phonemes was found to be statistically different from the normal distribution $(p<.005$, Table 2$)$. However, a visual inspection of the histogram (Figure 2) did not suggest significant gross violation.

The MCC dataset has 36 cases of missing items. We explored the missing data mechanism by conducting an independent-samples t-test, in which we compared items with missing values on MCC vs. items with observed values on MCC in terms of the remaining variables, namely, lexical frequency, AOA, number of phonemes, and item difficulty. As seen in Table 3, unlike lexical frequency, AOA, and item difficulty, when comparing the average number of phonemes across items with and without MCC values, Levine's test was statistically significant, $\mathrm{t}(42.54)=-5.34, p<.005$. However, regardless of whether equal variances were being assumed or not, there are significant differences between items with missing MCC values and those without missing MCC values across all variables (i.e. lexical frequency, $t(173)=$ $5.644, p<.005$, AOA, $t(173)=-3.629, p<.001$, number of phoneme, $t(173)=-5.335, p<.005$, and item difficulty, $t(173)=-6.253, p<.005)$. This result suggests that the missingness is not at random. 


\section{Main Analysis}

Multiple linear regression was used to test the predictive power of the model of three psycholinguistic variables, including AOA, lexical frequency, and word length, when added a fourth predictor, MCC. In this analysis, data were available for 139 items, due to the missingness of MCC values, as opposed to 175 items in the original model (Fergadiotis et al., 2015). Thus, the basic descriptive statistics and correlations between variables have changed, as demonstrated in Table 4. Both models were statistically significant $(p<.01)$. Specifically, model A explained $51.5 \%$ of the variance $(F(3,135)=47.75, p<.01)$, while model 2 accounted for $52.3 \%$ of the variance $(F(4,134)=36.76, p<.01)$. Indeed, there was a slight increase in the predictive power of model B compared to model A. However, the change was not statistically significant, $p=.13$ (Table 5). This can be explained by the statistically insignificant contribution of the MCC variable in the predictive power of model B $(p=.13)$, as indicated in Table 6. In the same table, according to the standardized coefficients, AOA was the most powerful predictor, followed by number of phonemes and lexical frequency. Particularly, a one-year increase of AOA, as shown under unstandardized coefficients, was associated with an increase of .19 units in item difficulty, holding the other predictors constant.

\section{Discussion}

In this study, we examined the predictive utility of MCC in estimating item difficulty parameters. Precise estimation of item difficulty parameters allows for increased efficacy and clinical usage of CNTs across settings and courses of treatment. In previous studies, a set of three psycholinguistic variables, AOA, lexical frequency and word length, was identified to serve this purpose. Because of MCC's nature to explain the interlayered relationships, specifically the word-word similarities in the semantic network, among words in the human lexicon, we 
hypothesized that adding MCC to the model can increase the predictive utility of item difficulty parameters. In this regard, we ran a multiple regression analysis to understand the contribution of MCC in estimating item difficulty parameters, along with the remaining three variables. Specifically, the explanatory power of two models, one with and one without MCC, were compared. As a result, the analysis suggested that, although the model with all four predictors accounted for more variance than the model with only the three original predictors, the change was not statistically significant and did not explain a significant amount of variance above and beyond the rest of the predictors in the model.

Our findings did not support our hypothesis, according to which MCC would further increase the explanatory power of the predictive model using traditional psycholinguistic variables (i.e., word length, AOA, and lexical frequency). In Castro and Stella's work (2019), MCC was a significant predictor of picture naming performance, even when accounting for the influence of other psycholinguistic variables and group differences $\left(\triangle R^{2}=.214\right)$. In both their study and our analysis, the coefficient for MCC was greater than both word length and lexical frequency, and slightly less than the coefficient for AOA, again, confirming that MCC was an indicative measure for lexical retrieval. Moreover, our result specified a strong negative correlation between MCC and item difficulty $(r=-.59)$, indicating that words with higher MCC were less difficult to be retrieved. This is consistent with Castro and Stella who argued that words with high closeness centrality were more likely to be produced than words with low closeness centrality. However, MCC correlated not only with the dependent variable, item difficulty parameter, but also with the other predictors. Our result showed a high correlation between MCC and lexical frequency $(r=.72)$ and a moderate correlation with number of phonemes $(r=-.64)$. Following this, the partial correlations between MCC and item difficulty 
was reduced to -.13 once the correlations among predictors were taken into account (Table 6). Hence, it is safe to say that MCC did not add as much unique information to the model as we had expected and thus, its contribution to the psycholinguistic model was not statistically significant, despite the high correlation with item difficulty.

\section{Post Hoc Analysis of Missingness}

There was a significant amount of missing values from the MCC dataset, with 36 missing cases in a total of 175 items. Importantly, the missingness was not at random. In addition to that, SPSS, within which we did our analysis, uses listwise deletion as a default choice. Subsequently, the items that had missing values for MCC were taken out of the analysis, meaning data on word length, AOA and lexical frequency of these items were also omitted. This resulted in an incomplete dataset compared to the one used by Fergadiotis and his colleagues when they built their 2015 model, introducing the potential for biased parameter estimation. Therefore, in order to evaluate the extent to which the missingness affects our analysis, a post hoc study was conducted to replicate the 2015 model. Specifically, we wanted to compare the explanatory power of our model, with the incomplete dataset, and the one with complete dataset. If missingness was not having a negative effect, the result would look similar to the 2015 study results. As shown in Table 6 and 7, our regression equations, even one that only consists of the original 3 variables, is distorted compared to the one obtained with full data. In other words, the model that reflected the missingness of MCC would have a lower explanatory power than which with the complete dataset, as reported in Fergadiotis et al. (2015) $\left(\operatorname{adj} . R^{2}=.50\right.$ and .62 , respectively). Therefore, given the missingness pattern, our results have to be interpreted very cautiously. 
Castro \& Stella's work (2019), from whom the MCC parameters in this paper were retrieved, recognized that 33 items were not found in the multiplex lexical network and thus, left them out of the analysis. They indicated that these missing words shared two mutual characteristics. They were either nouns that specifically used for types of animal or types of tool, or they had significantly low word frequency. Following that, to explore the influence of the missingness, the authors randomly removed 33 words from the 142 tested words, performed the logistic regression over the remaining items and repeated the random elimination of items for another 20 times, followed by the same analysis. They concluded that the average outcome was similar to the results they obtained from their main analysis, $(\mathrm{R} 2=0.2144 \pm 0.006)$, and concluded that the missingness did not affect their analysis.

Contradictorily, our exploration of missingness led us to an opposite conclusion, that the missing data mechanism was not benign. In Castro and Stella's approach, when the elimination of the 33 items was generated randomly, the missingness mechanism was assumed to be missing at random. This did not add up to the trends they found for the missing items, as provided above. In addition, when this approach was to be carried out, the subsets of the remaining data, on average, would always give the same solution as the full dataset, as long as a random sampling algorithm was employed. On the other hand, according to our post hoc analysis, when using the same predictors as Fergadiotis et al. (2015), for the incomplete dataset, the $R^{2}$ was significantly lower than the one reported in 2015.

\section{Future Direction}

We concluded that MCC did not uniquely contribute to the predictive utility of the algorithmic of item difficulty. However, due to the missing not at random mechanism in the MCC variables, a complete dataset of MCC values is needed to accurately evaluate the 
predictive utility of the model. This should be an area of research in future studies. In the case that generating MCC variables for all 175 PNT items is yet to be achievable, we suggest taking into account the missing data theory so as to eliminate any potential pattern in the missingness. If this were to work, it would result in a more refined item difficulty parameters prediction and further the study of computerized adaptive version of the CNTs. 


\section{References}

Ayala, R. J. de. (2013). The Theory and Practice of Item Response Theory. Guilford Publications.

Brady, M. C., Kelly, H., Godwin, J., Enderby, P., \& Campbell, P. (2016). Speech and language therapy for aphasia following stroke. The Cochrane Database of Systematic Reviews, 6, CD000425. https://doi.org/10.1002/14651858.CD000425.pub4

Brysbaert, M., \& New, B. (2009). Moving beyond Kučera and Francis: A critical evaluation of current word frequency norms and the introduction of a new and improved word frequency measure for American English. Behavior Research Methods, 41(4), 977-990. https://doi.org/10.3758/BRM.41.4.977

Castro, N., \& Stella, M. (2019). The multiplex structure of the mental lexicon influences picture naming in people with aphasia. https://doi.org/10.1093/comnet/cnz012

Cossa, F. M., Fabiani, M., Farinato, A., Laiacona, M., \& Capitani, E. (1999). The "preliminary neuropsychological battery". An instrument to grade the cognitive level of minimally responsive patients. Brain Injury, 13(8), 583-592.

https://doi.org/10.1080/026990599121322

Dell, G. S. (1986). A spreading-activation theory of retrieval in sentence production. Psychological Review, 93(3), 283.

Dell, G., Schwartz, M., Martin, N., Saffran, E., \& Gagnon, D. (1997). Lexical Access in Aphasic and Nonaphasic Speakers. Psychological Review, 104, 801-838. https://doi.org/10.1037/0033-295X.104.4.801

Druks, J., \& Masterson, J. (2000). An Object and Action Naming Battery. Journal of Neurolinguistics. https://doi.org/10.1037/t41579-000 
Embretson, S. E., \& Reise, S. P. (2000). Item response theory for psychologists (pp. xi, 371).

Lawrence Erlbaum Associates Publishers.

Empirical Evaluation of Computer-Adaptive Alternate Short Forms for the Assessment of Anomia Severity | Journal of Speech, Language, and Hearing Research. (n.d.). Retrieved November 19, 2020, from https://pubs.asha.org/doi/abs/10.1044/2019_JSLHR-L-19-0213

Fergadiotis, G., Hula, W. D., Swiderski, A. M., Lei, C.-M., \& Kellough, S. (2019). Enhancing the Efficiency of Confrontation Naming Assessment for Aphasia Using Computer Adaptive Testing. Journal of Speech, Language, and Hearing Research: JSLHR, 62(6), 1724-1738. https://doi.org/10.1044/2018_JSLHR-L-18-0344

Fergadiotis, G., Kellough, S., \& Hula, W. D. (2015). Item Response Theory Modeling of the Philadelphia Naming Test. Journal of Speech, Language \& Hearing Research, 58(3), 865-877. https://doi.org/10.1044/2015_JSLHR-L-14-0249

Fergadiotis, G., Swiderski, A., \& Hula, W. D. (2019). Predicting confrontation naming item difficulty. Aphasiology, 33(6), 689-709. https://doi.org/10.1080/02687038.2018.1495310

Fergadiotis, G., \& Wright, H. H. (2016). Modelling confrontation naming and discourse performance in aphasia. Aphasiology, 30(4), 364-380. https://doi.org/10.1080/02687038.2015.1067288

Fridriksson, J., Morrow-Odom, K. L., Moser, D., Fridriksson, A., \& Baylis, G. (2006). Neural recruitment associated with anomia treatment in aphasia. NeuroImage, 32, 1403-1412. https://doi.org/10.1016/j.neuroimage.2006.04.194

Goodglass, H., \& Kaplan, E. (1972). Boston Diagnostic Aphasia Examination, 3rd Edition | BDAE-3. https://www.parinc.com/Products/Pkey/16

Goodglass, H., \& Wingfield, A. (1997). Anomia: Neuroanatomical and cognitive correlates. 
Hula, W. D., Fergadiotis, G., Swiderski, A. M., Silkes, J. P., \& Kellough, S. (2020). Empirical Evaluation of Computer-Adaptive Alternate Short Forms for the Assessment of Anomia Severity. Journal of Speech, Language, and Hearing Research, 63(1), 163-172. https://doi.org/10.1044/2019_JSLHR-L-19-0213

Hula, W. D., Kellough, S., \& Fergadiotis, G. (2015). Development and Simulation Testing of a Computerized Adaptive Version of the Philadelphia Naming Test. Journal of Speech, Language, and Hearing Research: JSLHR, 58(3), 878-890. https://doi.org/10.1044/2015_JSLHR-L-14-0297

Kaplan, E., Goodglass, H., Weintraub, S., \& Goodglass, H. (1983). Boston naming test. Lea \& Febiger.

Kendall, D. L., Oelke Megan, Brookshire Carmel Elizabeth, \& Nadeau Stephen E. (2015). The Influence of Phonomotor Treatment on Word Retrieval Abilities in 26 Individuals With Chronic Aphasia: An Open Trial. Journal of Speech, Language, and Hearing Research, 58(3), 798-812. https://doi.org/10.1044/2015_JSLHR-L-14-0131

Kertesz, A. (2007). WAB-R: Western Aphasia Battery-Revised (Revised.). PsychCorp.

Kiran, S., Cherney, L., Kagan, A., Haley, K., Antonucci, S., Schwartz, M., Holland, A., \& Simmons-Mackie, N. (2018). Aphasia assessments: A survey of clinical and research settings. Aphasiology, 32, 47-49. https://doi.org/10.1080/02687038.2018.1487923

Kittredge, A. K., Dell, G. S., Verkuilen, J., \& Schwartz, M. F. (2008). Where is the effect of frequency in word production? Insights from aphasic picture naming errors. Cognitive Neuropsychology, 25(4), 463-492. https://doi.org/10.1080/02643290701674851 
Kuperman, V., Stadthagen-Gonzalez, H., \& Brysbaert, M. (2012). Age-of-acquisition ratings for 30,000 English words. Behavior Research Methods, 44(4), 978-990. https://doi.org/10.3758/s13428-012-0210-4

Levelt, W. J. M., Roelofs, A., \& Meyer, A. S. (1999). A theory of lexical access in speech production. BEHAVIORAL AND BRAIN SCIENCES, 76.

Lord, F. M., Novick, M. R., \& Birnbaum, A. (1968). Statistical theories of mental test scores. Addison-Wesley.

Newman, M. E. J. (2010). Networks: An introduction. Oxford University Press.

Quique, Y. M., Evans, W. S., \& Dickey, M. W. (2019). Acquisition and Generalization Responses in Aphasia Naming Treatment: A Meta-Analysis of Semantic Feature Analysis Outcomes. American Journal of Speech-Language Pathology, 28(1S), 230-246. https://doi.org/10.1044/2018_AJSLP-17-0155

Raymer, A. M., \& Rothi, L. J. G. (2012). Impairments of word comprehension and production. In R. Chapey (Ed.), Language intervention strategies in aphasia and related neurogenic communication disorders (5th ed., pp. 607-631). Lippincott Williams \& Wilkins.

Roach, A., Schwartz, M., Martin, N., Grewal, R., \& Brecher, A. (1996). The Philadelphia Naming Test: Scoring and rationale. Clin. Aphasiol., 24.

Schwartz, M. F., Dell, G. S., Martin, N., Gahl, S., \& Sobel, P. (2006). A case-series test of the interactive two-step model of lexical access: Evidence from picture naming. Journal of Memory and Language, 54(2), 228-264.

Schwartz, M. F., Kimberg, D. Y., Walker, G. M., Faseyitan, O., Brecher, A., Dell, G. S., \& Coslett, H. B. (2009). Anterior temporal involvement in semantic word retrieval: Voxel- 
based lesion-symptom mapping evidence from aphasia. Brain: A Journal of Neurology, 132(Pt 12), 3411-3427. https://doi.org/10.1093/brain/awp284

Snodgrass, J. G., \& Vanderwart, M. (1980). A standardized set of 260 pictures: Norms for name agreement, image agreement, familiarity, and visual complexity. Journal of Experimental Psychology: Human Learning and Memory, 6(2), 174-215. https://doi.org/10.1037/02787393.6.2.174

Swinburn, K., Howard, D., \& Porter, G. (2004). CAT: Comprehensive aphasia test. Psychology Press.

Walker, G. M., \& Schwartz, M. F. (2012). Short-form Philadelphia naming test: Rationale and empirical evaluation. American Journal of Speech-Language Pathology, 21(2). https://link.galegroup.com/apps/doc/A290112849/PPNU?sid=lms 


\section{Tables}

Table 1

Correlations between the psycholinguistics variables

\begin{tabular}{lccccc}
\hline Variables & 1 & 2 & 3 & 4 & 5 \\
\hline 1. Multiplex closeness centrality & 0.001 & & & & \\
2. Lexical frequency & $.72^{* *}$ & 0.29 & & & \\
3. Age of acquisition & $-.40^{* *}$ & $-.50^{* *}$ & 1.8 & & \\
4. Number of phonemes & $-.64^{* *}$ & $-.50^{* *}$ & $.38^{* *}$ & 3.14 & \\
5. Item difficulty & $-.59^{* *}$ & $-.62^{* *}$ & $.64^{* *}$ & $.63^{* *}$ & 0.56 \\
M & 0.34 & 2.7 & 4.95 & 4.5 & -0.45 \\
SD & 0.02 & 0.53 & 1.34 & 1.77 & 0.75 \\
\hline
\end{tabular}

**. Correlation is significant at the 0.01 level (2-tailed). 


\section{Table 2}

Tests of Normality

\begin{tabular}{lcccccc}
\hline & \multicolumn{3}{c}{ Kolmogorov-Smirnov ${ }^{\mathrm{a}}$} & \multicolumn{3}{c}{ Shapiro-Wilk } \\
\cline { 2 - 7 } & Statistic & $d f$ & $p$ & Statistic & $d f$ & $p$ \\
\hline Multiplex Closeness Centrality & 0.08 & 139 & 0.05 & 0.97 & 139 & 0.004 \\
Lexical Frequency & 0.04 & 139 & $.200^{*}$ & 0.99 & 139 & 0.536 \\
Age of Acquisition & 0.07 & 139 & $.200^{*}$ & 0.97 & 139 & 0.005 \\
Number of Phonemes & 0.2 & 139 & 0 & 0.89 & 139 & 0 \\
Item Difficulty & 0.01 & 139 & 0.045 & 0.98 & 139 & 0.116 \\
\hline
\end{tabular}

*. This is a lower bound of the true significance.

a. Lilliefors Significance Correction 
Table 3

Independent-Samples T-test

\begin{tabular}{|c|c|c|c|c|c|c|c|}
\hline & \multirow[b]{2}{*}{$t$} & \multirow[b]{2}{*}{$d f$} & \multirow[b]{2}{*}{$p$} & \multirow{2}{*}{$\begin{array}{c}\text { Mean } \\
\text { Difference }\end{array}$} & \multirow{2}{*}{$\begin{array}{l}\text { Std. Error } \\
\text { Difference }\end{array}$} & \multicolumn{2}{|c|}{$\begin{array}{l}95 \% \text { Confidence } \\
\text { Interval of the } \\
\text { Difference }\end{array}$} \\
\hline & & & & & & Lower & Upper \\
\hline Lexical Frequency & 5.64 & 173 & $<.001$ & .52 & .09 & .34 & .7 \\
\hline Age of Acquisition & -3.63 & 173 & $<.001$ & -.89 & .24 & -1.36 & -.4 \\
\hline Number of Phonemes & -5.34 & 42.54 & $<.001$ & -2.03 & .38 & -2.8 & -1.26 \\
\hline Item Difficulty & -6.25 & 173 & $<.001$ & -.79 & .12 & -1.04 & -.54 \\
\hline
\end{tabular}

Note. Levene's tests were not significant for any of the variables with the exception of number of phonemes, $F(2,173)=16.67, p<.001$. For number of phonemes, the adjusted degrees of freedom are reported. 
Table 4

Correlations Between the Psycholinguistic Variables

\begin{tabular}{lccccc}
\hline & 1 & 2 & 3 & 4 & 5 \\
\hline 1. Multiplex Closeness Centrality & & & & & \\
2. Lexical Frequency & $.72 *$ & & & & \\
3. Age of Acquisition & $-.40^{*}$ & $-.43^{*}$ & & & \\
4. Number of Phonemes & $-.64^{*}$ & $-.44^{*}$ & $.26^{*}$ & & \\
5. Item Difficulty & $-.59^{*}$ & $-.55^{*}$ & $.56^{*}$ & $.53^{*}$ & \\
$M$ & .33 & 2.81 & 4.76 & 4.07 & -.61 \\
$S D$ & .02 & .5 & 1.25 & 1.37 & .66 \\
\hline
\end{tabular}


Table 5

Model Summary

\begin{tabular}{ccccccccc}
\hline \multirow{2}{*}{ Model } & \multirow{2}{*}{$R^{2}$} & \multirow{2}{*}{ Adj. $R^{2}$} & \multirow{2}{*}{$F$} & $p$ & \multicolumn{4}{c}{ Change Statistics } \\
\cline { 6 - 9 } & & & & $\Delta F$ & $d f 1$ & $d f 2$ & $p$ \\
\hline Model A & 0.51 & 0.5 & 47.75 & $<.001$ & 47.75 & 3 & 135 & $<.001$ \\
Model B & 0.52 & 0.51 & 36.76 & $<.001$ & 2.35 & 1 & 134 & 0.13 \\
\hline
\end{tabular}

Note. Model A includes Number of Phonemes, Age of Acquisition, and Lexical Frequency.

Model B includes Number of Phonemes, Age of Acquisition, Lexical Frequency, and

Multiplex Closeness Centrality. 
Table 6

Model Coefficients

\begin{tabular}{|c|c|c|c|c|c|c|c|c|}
\hline \multirow[b]{2}{*}{ Model } & \multicolumn{3}{|c|}{ Unstandardized Coefficients } & \multicolumn{3}{|c|}{ Standardized Coefficients } & \multicolumn{2}{|c|}{ Correlations } \\
\hline & $b$ & $S E$ & $95 \% C I$ & $\beta$ & $t$ & $p$ & Zero-order & Partial \\
\hline 1 (Constant) & -1.21 & 0.41 & {$[-2.03,-.40]$} & & -2.96 & 0.004 & & \\
\hline Lexical Frequency & -0.33 & 0.09 & {$[-.52,-.15]$} & -0.26 & -3.57 & 0.000 & & \\
\hline Age of Acquisition & 0.19 & 0.04 & {$[.12, .26]$} & 0.36 & 5.45 & 0.000 & & \\
\hline Number of Phonemes & 0.15 & 0.03 & {$[.09, .22]$} & 0.32 & 4.76 & 0.000 & & \\
\hline 2 (Constant) & 0.14 & 0.97 & {$[-1.78,2.07]$} & & 0.15 & 0.884 & & \\
\hline Lexical Frequency & -0.23 & 0.12 & {$[-.46, .00]$} & -0.17 & -1.96 & 0.052 & -0.55 & -0.17 \\
\hline Age of Acquisition & 0.19 & 0.04 & {$[.12, .26]$} & 0.35 & 5.27 & 0.000 & 0.56 & 0.41 \\
\hline Number of Phonemes & 0.12 & 0.04 & {$[.05, .20]$} & 0.26 & 3.30 & 0.001 & 0.53 & 0.27 \\
\hline Multiplex Closeness Centrality & -4.44 & 2.89 & {$[-10.16,1.28]$} & -0.16 & -1.53 & 0.127 & -0.59 & -0.13 \\
\hline
\end{tabular}

a. Dependent Variable: Item Difficulty 
Table 7

Coefficients $^{a}$

\begin{tabular}{|c|c|c|c|c|c|}
\hline \multirow[b]{2}{*}{ Variables } & \multicolumn{2}{|c|}{$\begin{array}{c}\text { Complete Dataset } \\
\text { (Fergadiotis et al., 2015) }\end{array}$} & \multicolumn{3}{|c|}{ Incomplete Dataset } \\
\hline & $b$ & $S D$ & $b$ & $S D$ & $95 \% C I$ \\
\hline (Constant) & -1.22 & .35 & -1.21 & .41 & {$[-2.03,-.40]$} \\
\hline Lexical Frequency & -.36 & .08 & -.33 & .09 & {$[-.52,-.15]$} \\
\hline Age of Acquisition & .21 & .03 & .19 & .04 & {$[.12, .26]$} \\
\hline Number of Phonemes & .15 & .02 & .15 & .03 & {$[.09, .22]$} \\
\hline
\end{tabular}

a. Dependent Variable: Item Difficulty 


\section{Figures}

\section{Figure 1}

Changes in Item Difficulty as a Function of the Four Psycholinguistic Variables
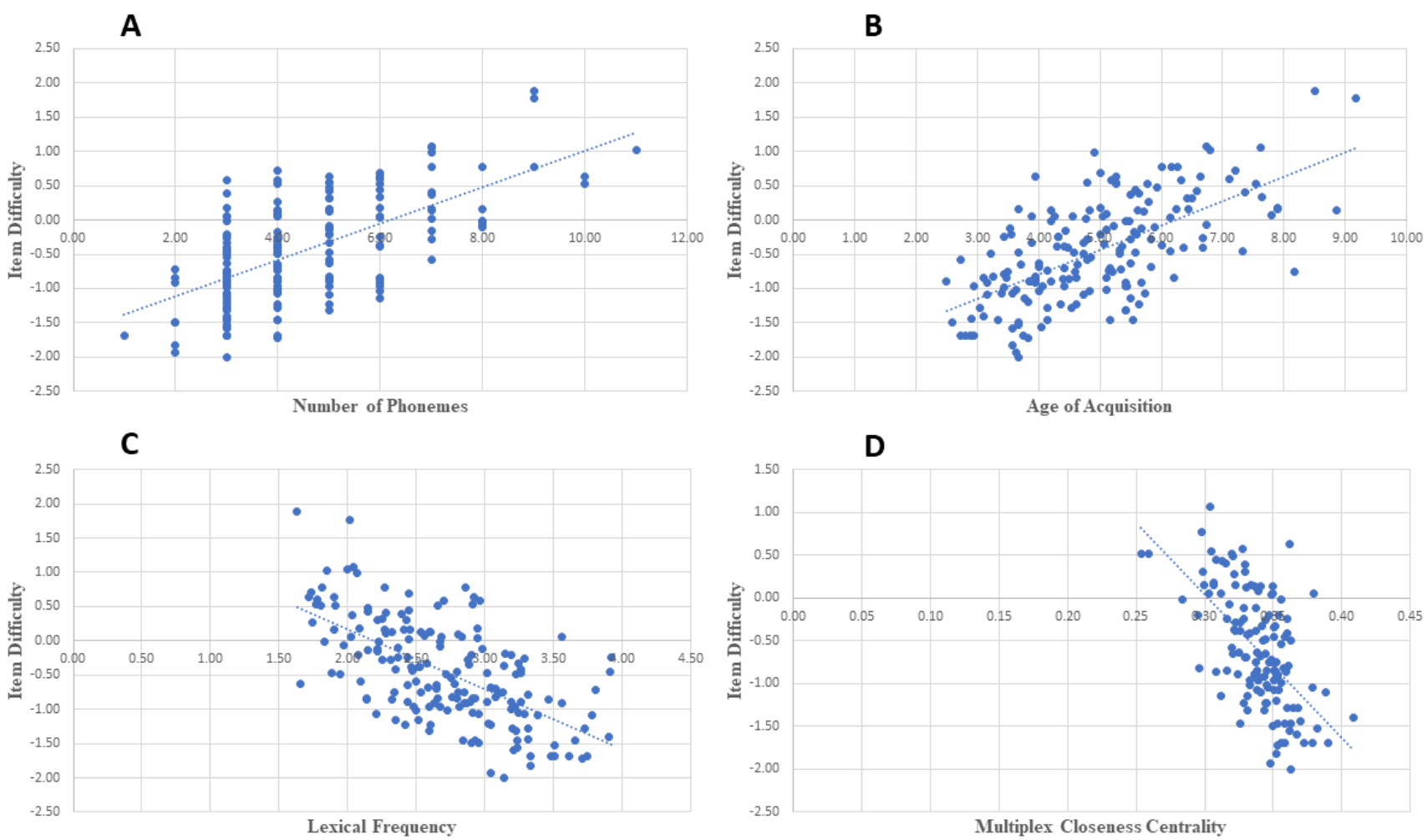


\section{Figure 2}

The Distribution of the Four psycholinguistic Variables
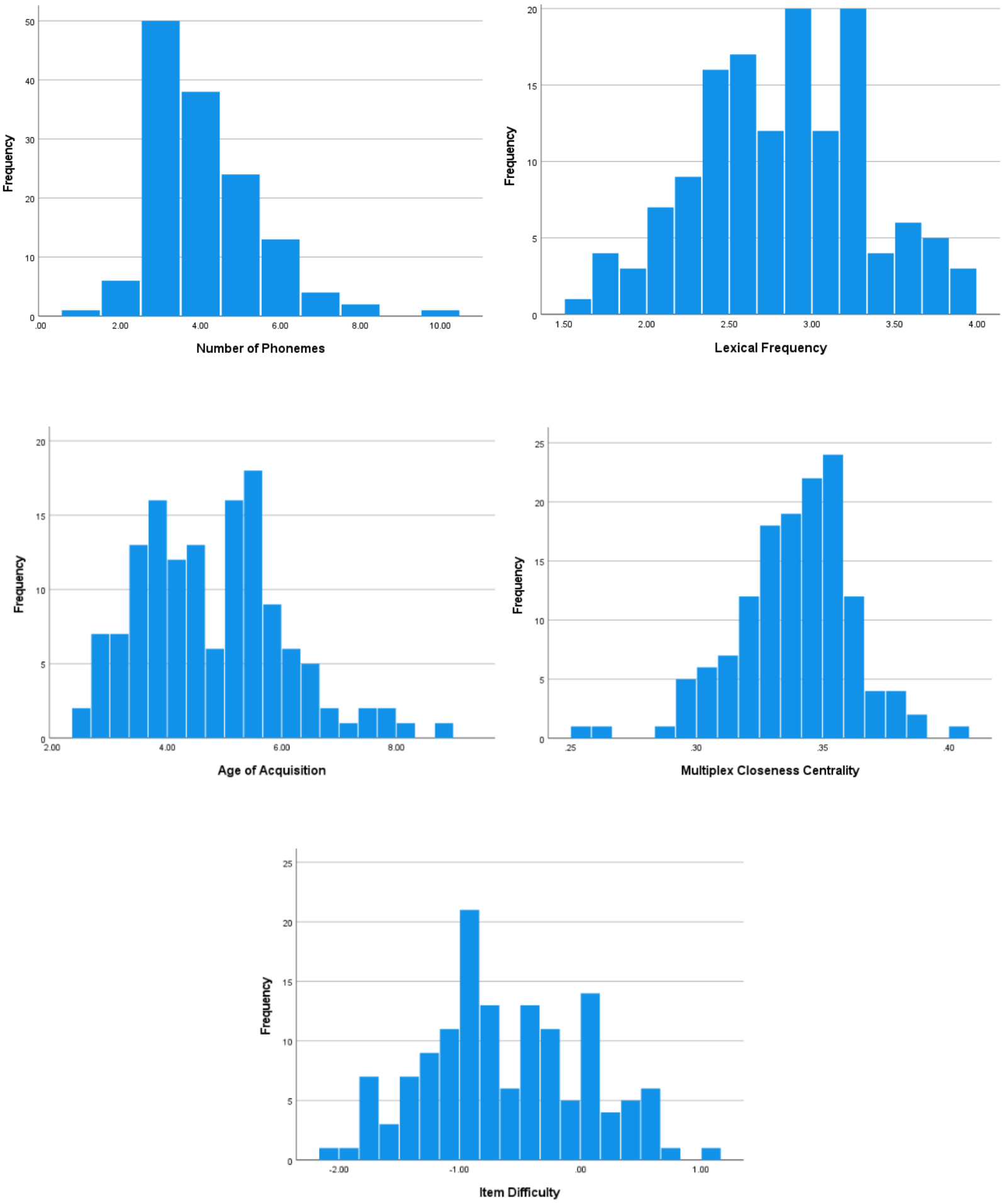